\title{
EL ARBITRAJE DE INVERSIONES ENTRE LA UNIÓN EUROPEA Y SINGAPUR: ¿DEL ARBITRAJE DE DIFERENCIAS ESTADO-INVERSOR A UN SISTEMA DE TRIBUNALES DE INVERSIONES?*
}

\author{
Investment arbitration between the European Union \\ and Singapore: from the Investor-State Dispute Settlement \\ to an Investment Court System?
}

\author{
Sophie Recio San Emeterio \\ Universidad de Deusto \\ sophie.recio@opendeusto.es
}

http://dx.doi.org/10.18543/ed-67(1)-2019pp353-383

Recibido: 10.02.2019

Aceptado: 21.06.2019

\section{Resumen}

El régimen de arbitraje de inversiones entre la Unión Europea (UE) y Singapur ha pasado del arbitraje de diferencias Estado-inversor propuesto por el borrador del Acuerdo de Libre Comercio entre la UE y Singapur del 2015 a un Sistema de Tribunales de Inversiones en el borrador del Acuerdo de Protección de las Inversiones entre la UE y Singapur (APIUES) del 2018. Esta evolución se fundamenta en: a) los cambios de forma y fondo introducidos por el APIUES; y, b) un análisis comparativo

* Cómo citar / Citation 'Chicago-Deusto' (Autor-fecha / Author-date / Lista de referencias / Reference list entries): San Emeterio, Sophie Recio. 2019. «El arbitraje de inversiones entre la Unión Europea y Singapur: ¿Del arbitraje de diferencias Estado-Inversor a un sistema de Tribunales De Inversiones?». Estudios de Deusto 67, n. ${ }^{\circ} 1$ : 353-383. http:// dx.doi.org/10.18543/ed-67(1)-2019pp353-383. 
de los sistemas de tribunales de inversiones previstos en el APIUES, la Asociación Transatlántica para el Comercio y la Inversión entre la UE y los Estados Unidos, y el Acuerdo Económico y Comercial Global entre la UE y Canadá.

\title{
Palabras clave
}

Arbitraje; Unión Europea; Singapur; Sistema de Tribunales de Inversiones; Asociación Transatlántica para el Comercio y la Inversión; Acuerdo Económico y Comercial Global.

\begin{abstract}
The investment arbitration regime between the European Union (EU) and Singapore has gone from the Investor-State Dispute Settlement mechanism suggested in the 2015 draft of the EU-Singapore Free Trade Agreement to an Investment Court System in the 2018 draft of the EU-Singapore Investment Protection Agreement (EUSIPA). Such evolution is built upon: a) the changes in form and substance introduced by the EUSIPA; and, b) a comparative analysis of the Investment Court Systems foreseen for the EUSIPA, the Transatlantic Trade and Investment Partnership, and the EU-Canada Comprehensive Economic and Trade Agreement.
\end{abstract}

\section{Keywords}

Arbitration; European Union; Singapore; Investment Court System; TTIP; CETA. 


\begin{abstract}
SUMARIO: I. INTRODUCCIÓN. II. CONTEXTUALIZACIÓN DEL ACUERDO DE LIBRE COMERCIO ENTRE LA UNIÓN EUROPEA Y SINGAPUR. III. EL RÉGIMEN DE ARBITRAJE DE INVERSIONES EN EL ACUERDO DE LIBRE COMERCIO ENTRE LA Unión EuROPEA Y Singapur DEL 2015. 1. Terminación de los Acuerdos sobre Promoción y Protección Recíproca de las Inversiones y las sunset clauses. 2. Remisión a reglas internacionales. 3. Regulación de las consultas. 4. Adopción del no U-turn. IV. EL RÉGIMEN DE ARBITRAJE DE INVERSIONES EN EL ACUERDO DE PROTECCIÓN DE LAS INVERSIONES ENTRE LA UNIÓN EUROPEA Y SINGAPUR DEL 2018. 1. Indicios de un Sistema de Tribunales de Inversiones. 2. Sobre las disposiciones derivadas de reglas internacionales. 3. Sobre la regulación de las consultas. 4. Sobre la adopción del no U-turn. V. OBSTÁCULOS LEGALES AL ACUERDO DE LIBRE COMERCIO ENTRE LA UNIÓN EUROPEA Y SingapUR. 1. El artículo 59 del Convenio de Viena sobre el Derecho de los Tratados y el Dictamen 2/15. 2. La Sentencia de la Gran Sala del Tribunal de Justicia de la UE, de 6 de marzo de 2018, sobre el caso Achmea. VI. CONCLUSIONES. VII. BIBLIOGRAFÍA.
\end{abstract}

\title{
I. INTRODUCCIÓN
}

Los Acuerdos de Promoción y Protección Recíproca de Inversiones (APPRIs) son práctica corriente en el ámbito europeo e internacional desde el Tratado Bilateral de Inversión (TBI) entre Alemania y Pakistán de 1959. En sus primeros años, estos acuerdos no regulaban el arbitraje de diferencias Estado-inversor (mejor conocido como Investor-State Dispute Settlement o ISDS), un mecanismo legal que permite a un inversor dirigirse contra un Estado en el que ha realizado inversiones mediante un procedimiento arbitral ${ }^{1}$. La ausencia de un claro marco legal resultaba problemática pues hacía proliferar el uso de procedimientos paralelos, i.e. el empleo simultáneo de la vía judicial y la vía arbitral por los inversoresdemandantes ${ }^{2}$.

El arbitraje de diferencias Estado-inversor sólo se institucionalizaría con el Convenio sobre la Resolución de Disputas de Inversión entre Estados y Nacionales de otros Estados, celebrado en Washington en 1965. El conocido como Convenio de Washington marcó un punto de inflexión en el ámbito del

${ }^{1}$ Marta Latek, Investor-State Dispute Settlement (ISDS) State of play and prospects for reform (Servicios De Estudios Del Parlamento Europeo (EPRS), 2014), edición en PDF, 2.

2 Advisory Council on International Affairs, «Introduction», International Investment Dispute Settlement, n. 95 (La Haya: Advisory Council on International Affairs, 2015), 7. 
arbitraje internacional, pues sentó las bases para la creación del Centro Internacional de Arreglo de Diferencias relativas a Inversiones (CIADI) del Banco Mundial, cuyas Reglas Procesales Aplicables a los Procedimientos de Arbitraje (Reglas de Arbitraje) serían a su vez retomadas en 1976 por la Comisión de las Naciones Unidas para el Derecho Mercantil Internacional (CNUDMI) ${ }^{3}$. Hoy en día, el CIADI y la CNUDMI son los órganos de referencia para el arbitraje de diferencias Estado-inversor. De hecho, todos los Estados Miembros de la Unión Europea (UE) salvo Polonia han ratificado el Convenio de Washington. En el caso de la UE, no ha podido adherirse al CIADI por no ser un estado conforme requiere el artículo 67 del Convenio ${ }^{4}$.

A nivel de la UE, el arbitraje de inversiones ha evolucionado en los últimos cinco años del tradicional régimen de arbitraje de diferencias Estadoinversor a un Sistema de Tribunales de Inversiones (STI) recogido en distintos Acuerdos de Libre Comercio (ALCs) entre la UE y sus socios comerciales. En particular, destacan la Asociación Transatlántica para el Comercio y la Inversión (ATCI) entre la UE y Estados Unidos (EEUU), mejor conocida como Transatlantic Trade and Investment Partnership o TTIP, y el Acuerdo Económico y Comercial Global (AECG) entre la UE y Canadá, mejor conocido como Comercial Global o Comprehensive Economic and Trade Agreement (CETA).

En el 2017, se anunció que el STI se incorporaría también al borrador del Acuerdo de Libre Comercio entre la UE y Singapur (ALCUES) del 2015, siendo necesario para ello reformar el Capítulo IX sobre arbitraje de diferencias Estado-inversor del acuerdo. A continuación, el 18 de abril del 2018, se reformó y dividió el ALCUES del 2015 en otros dos acuerdos: a) el Acuerdo de Protección de las Inversiones entre la UE y Singapur (APIUES), dedicado exclusivamente al ámbito de las inversiones y al arbitraje sobre las mismas; $y, b)$ una nueva versión del ALCUES, dedicada principalmente al comercio de bienes y servicios, derechos de propiedad intelectual, energías renovables, etc $^{5}$. A pesar del anuncio del 2017, ni el APIUES ni el ALCUES del 2018 mencionan en ningún momento la aplicación de un STI. En consecuencia, esta investigación tiene por objetivo analizar la evolución del borrador del ALCUES del 2015 hasta el borrador del APIUES del 2018 para determinar si, efectivamente, se ha producido ese cambio en el régimen de arbitraje de

\footnotetext{
3 Advisory Council on International Affairs. «Introduction», 7-8.

${ }^{4}$ Convenio de Washington, de 18 de marzo de 1965, sobre Arreglo de Diferencias Relativas a Inversiones entre Estados y nacionales de otros Estados $\left(B O E \mathrm{n}^{\circ}{ }^{\circ} 219\right.$ de 13 de septiembre de 1994).

${ }_{5}$ «Guide to the EU-Singapore Free Trade Agreement and Investment Protection Agreement», Comisión Europea, acceso el 19 de abril de 2018, http://trade.ec.europa.eu/ doclib/docs/2018/april/tradoc_156711.pdf.
} 
inversiones. Hemos elegido el APIUES en vez del nuevo borrador del ALCUES para analizar el régimen de arbitraje a fecha del 2018 en base de la mayor especialidad de este primer acuerdo, pues regula exclusivamente la protección de las inversiones.

En cuanto al acuerdo entre la UE y Singapur (incluyendo tanto ALCUES como APIUES), son varias las razones que nos han llevado a seleccionar este tema de investigación. En primer lugar, el ALCUES es un acuerdo de gran relevancia doctrinal y jurisprudencial. En efecto, el borrador del 2015 ha planteado serias dudas en materia de Derecho de la Unión Europea (DUE), a las que el Tribunal de Justicia de la Unión (TJUE) ha dado respuesta en su Dictamen 2/15, de 16 de mayo del 2017, y en la Sentencia de 6 de marzo de 2018 sobre el caso Achmea (ver epígrafe V). En segundo lugar, se trata de un acuerdo de clara relevancia actual, como demuestra el hecho de tener un Dictamen y una Sentencia del TJUE, así como dos acuerdos totalmente nuevos, en poco más de un año.

Para analizar la evolución del arbitraje en el ALCUES-APIUES, se contextualizará en primer lugar el borrador del ALCUES del 2015. Así, se revisarán los intereses subyacentes de la UE y de Singapur en el acuerdo, el desarrollo de las negociaciones hasta llegar al borrador del APIUES, y los próximos movimientos institucionales de la Unión necesarios para la entrada en vigor del acuerdo. Establecido el marco general, se realizará después un análisis de fondo sobre el régimen de arbitraje de inversiones en la versión del ALCUES del 2015 y en el borrador del APIUES del 2018, siguiendo un orden cronológico.

Respecto del ALCUES del 2015, se explicará primero el funcionamiento del Acuerdo con respecto de otros APPRIs existentes entre la UE y Singapur, y después se analizarán las tres características principales del régimen de arbitraje de diferencias Estado-inversor previsto en este borrador. A continuación, para determinar si el borrador del APIUES del 2018 se distancia del borrador del ALCUES del 2015 y se encamina hacia la adopción de un STI, se realizará un estudio de doble nivel: a) se compararán las características propias del STI recogidas en la ATCI y el AECG con las disposiciones del APIUES; y, b) se estudiarán los principales cambios del APIUES respecto del régimen general de arbitraje de inversiones del ALCUES del 2015.

Siguiendo ese orden, comenzamos pues con la contextualización del ALCUES.

\section{CONTEXTUALIZACIÓN DEL ACUERDO DE LIBRE COMERCIO ENTRE LA UNIÓN EUROPEA Y SINGAPUR}

En abril del 2007, el Consejo de la UE autorizó el comienzo de las negociaciones para un Acuerdo Comercial Regional (ACR) entre la UE y la 
Asociación de Naciones del Sudeste Asiático (ASEAN) ${ }^{6}$. De haberse llevado a cabo, este acuerdo habría dado lugar a la creación de un ALC «megaregional», pues incorporaría los entonces 27 Estados Miembros de la Unión y los 10 de ASEAN (Brunei, Camboya, Filipinas, Indonesia, Laos, Malasia, Myanmar, Singapur, Tailandia y Vietnam) ${ }^{7}$.

Sin embargo, las negociaciones para un ACR UE-ASEAN fracasaron debido a la estructura de las negociaciones. El Consejo de la UE había autorizado las negociaciones «quedando sobreentendido que el objetivo era negociar un acuerdo de libre comercio de región a región $»^{8}$. Sin embargo, la ASEAN tiende a negociar individualmente, con un representante por cada Estado Miembro y otro por la organización en sí. En consecuencia, durante las negociaciones, cada área discutida debía ser negociada por la Unión con cada Estado Miembro de ASEAN y se ralentizaba la totalidad del proceso. Ante estas dificultades, ambas partes decidieron suspender las negociaciones UE-ASEAN en el $2009^{9}$ y la UE en particular cambió su estrategia hacia el sudeste asiático: en vez de negociar con ASEAN, la UE decidió lanzar negociaciones para ALCs bilaterales con cuatro países específicos (Malasia, Singapur, Tailandia y Vietnam) y empezar por Singapur ${ }^{10}$.

Este país del Sudeste asiático es un socio comercial fundamental para la UE. En lo relativo a inversiones, Singapur es el $8^{\circ}$ mayor recipiente de Inversión Extranjera Directa (IED) de la UE, con 154 mil millones de euros invertidos en Singapur en el 2017, y el $9^{\circ}$ mayor remitente de IED a la UE, con $59 €$ mil millones invertidos en la Unión en el mismo año ${ }^{11}$. A estas ventajas económicas se suma la experiencia de Singapur con los ALCs, destacando por ejemplo el Acuerdo de Libre Comercio entre los Estados Unidos y Singapur (ALCEUS) del 2005. Además, un ALC con Singapur resulta muy interesante para la UE en tanto que puede permitir

6 «Desclasificación parcial del documento 17494/09 RESTREINT UE», Consejo de la Unión Europea, acceso el 17 de abril de 2018, http://data.consilium.europa.eu/doc/document/ST-17494-2009-EXT-1/es/pdf.

7 Deborah Elms, «Understanding the EU-Singapore Free Trade Agreement», en Australia, the European Union and the New Trade Agenda, ed. por Annmarie Elijah, Donald Kenyon, Karen Hussey y Pierre Van Der Eng (Canberra: Australian National University Press, 2017), edición en PDF, 36-37.

8 «Desclasificación parcial del documento 17494/09 RESTREINT UE», Consejo de la Unión Europea, acceso el 17 de abril de 2018.

9 «Desclasificación parcial del documento 17494/09 RESTREINT UE», Consejo de la Unión Europea, acceso el 17 de abril de 2018.

${ }^{10}$ Elms, «Understanding the EU-Singapore Free Trade Agreement», 36-37.

11 «EU-Singapore Trade \& Investment», Comisión Europea, acceso el 19 de abril de 2018, https://eeas.europa.eu/sites/eeas/files/9045-trade-eu-singapore-trade-booklet-170630.pdf. 
crear un puente hacia un futuro acuerdo con ASEAN, tal y como planificado inicialmente ${ }^{12}$.

Impulsado por ese interés económico, el Consejo de la UE autorizó las negociaciones para el ALCUES en diciembre del $2009^{13}$ y éstas comenzaron en marzo del $2010^{14}$. Las negociaciones abarcaban ámbitos muy distintos: acceso al mercado, barreras comerciales, servicios, derechos de propiedad intelectual, competitividad, desarrollo sostenible y protección de las inversiones $^{15}$. La diversidad de áreas estudiadas, más allá de las tradicionales disposiciones sobre comercio de bienes y servicios, y la incorporación de un capítulo sobre inversiones hacían del ALCUES un acuerdo único: un ALC de «nueva generación» por la diversidad de temas cubiertos ${ }^{16} \mathrm{y}$ «el primer ALC de la UE con un Estado asiático que incorporaba un capítulo sobre inversiones» ${ }^{17}$. Más aún, el ALCUES marcaba la transición en los acuerdos de inversión de la UE de una «light touch regulation» a una «more comprehensive regulation», es decir una transición hacia acuerdos de inversión más detallados, claros y que aportaban mayor seguridad jurídica ${ }^{18}$. Debe señalarse aquí que la UE tiene capacidad para realizar acuerdos de inversiones gracias al artículo 207 del Tratado de Funcionamiento de la UE (TFUE), establecido por el Tratado de Lisboa del 2007 y que otorga competencia exclusiva a la UE sobre la política comercial común, incluyendo ello las IED ${ }^{19}$.

Las negociaciones del ALCUES acabaron en octubre del 2014. A continuación, en julio del 2015, la Comisión Europea (CE) pidió al Tribunal de

12 Elms, «Understanding the EU-Singapore Free Trade Agreement», 37 y 40.

${ }_{13}$ Marise Cremona, «Shaping EU Trade Policy post-Lisbon: Opinion 2/15 of 16 May 2017», en European Constitutional Law Review, ed. por Leonard F. M. Besselink, Monica Claes y Jan-Herman Reestman, Vol. 14 (Cambridge: Cambridge University Press, 2018), edición en PDF, 231-232.

${ }^{14}$ Locknie Hsu, «EU-ASEAN trade and investment relations with special focus on Singapore», en European Yearbook of International Economic Law, ed. por Christoph Herrmann, Markus Krajewski y Jörg Philipp Terhechte, Vol. 6 (Springer, 2015), edición en PDF, 238.

${ }^{15}$ Richard Allen, «EU Court thwarts prompt ratification of EU-Singapore Free Trade Agreement», en Global Arbitration News (2017).

16 «CJEU rules on EU's proposed free trade agreement with Singapore», Norton Rose Fulbright, acceso el 3 de abril de 2018, http://www.nortonrosefulbright.com/knowledge/ publications/149650/cjeu-rules-on-eus-proposed-free-trade-agreement-with-singapore.

${ }_{17} \mathrm{Hsu}$, «EU-ASEAN trade and investment relations with special focus on Singapore», 238, traducción de la autora.

${ }^{18}$ Steffen Hindelang y Carl-Philipp Sassenrath, The investment chapters of the EU'S international trade and investment agreements in a comparative perspective (Parlamento Europeo, Directorate-General for External Policies, 2015), edición en PDF, 11.

${ }_{19} \mathrm{Hsu}$, «EU-ASEAN trade and investment relations with special focus on Singapore», 236. 
Luxemburgo que se pronunciara sobre el reparto competencial entre la Unión y los Estados Miembros de las áreas abarcadas por el borrador y sobre la consiguiente naturaleza del ALCUES ${ }^{20}$. El TJUE dio respuesta a esta consulta en el conocido como Dictamen 2/15, de 16 de mayo del 2017, donde estableció que el borrador del ALCUES del 2015 tenía tanto materias que eran competencia exclusiva de la UE como materias que eran competencia compartida con los Estados Miembros y por lo tanto era un acuerdo de naturaleza mixta (ver epígrafe V.1) ${ }^{21}$.

Más tarde, el 4 de diciembre del 2017, representantes de Singapur y de la Comisión de Comercio Internacional del Parlamento Europeo (PE) se reunieron para revisar los avances del acuerdo y eventualmente anunciaron que «el arbitraje de diferencias Estado-inversor previsto en el Capítulo IX de ALCUES sería reemplazado por un mecanismo de STI $»^{22}$. Este cambio en el régimen de arbitraje del ALCUES estaba relacionado con la ATCI. Para calmar las críticas generalizadas sobre la falta de imparcialidad y predictibilidad del sistema de arbitraje de diferencias Estado-inversor en los ALCs de la UE, la CE propuso en el documento «Investment in TTIP and beyond - the path for reform - Enhancing the right to regulate and moving from current ad hoc arbitration towards an Investment Court» de mayo del 2015 sustituir el arbitraje de diferencias Estado-inversor por el STI en un primer momento y un Tribunal Multilateral de Inversiones posteriormente ${ }^{23}$. La ATCI y el AECG fueron los primeros acuerdos de la UE en incorporar el STI (ver epígrafe IV.1), anunciado luego en el 2017 para el ALCUES ${ }^{24}$.

${ }^{20}$ Edward Borovikov, Bogdan Evtimov y Anna Crevon-Tarassova, «European Union», en The International Arbitration Review, ed. por James H. Carter, n. ${ }^{\circ} 7$ (Londres: Law Business Research Ltd, 2016), edición en PDF, 188.

${ }^{21}$ Define Mariola Urrea Corres como acuerdo de naturaleza mixta aquel acuerdo para cuya reforma «interviene una institución de la Unión Europea, pero la vigencia de tal reforma está condicionada a la aprobación por los Estados miembros de conformidad con sus normas constitucionales», por oposición a un acuerdo de naturaleza especial o autónoma, «donde la competencia recae exclusivamente en las instituciones de la Unión» (Mariola Urrea Corres, «La política (común) de seguridad y defensa en el Tratado de Lisboa: La eficacia como objetivo, la flexibilidad como instrumento y la ambición como propuesta», en Revista Española de Derecho Europeo, n. ${ }^{\circ} 33$ (Pamplona: Editorial Civitas, SA, 2010), 4.

${ }^{22}$ Eirini Roussou, Free Trade Agreement between the EU and the Republic of Singapore-Analysis. (Parlamento Europeo, Directorate-General for External Policies, 2018), edición en PDF, 2.

23 «Investment in TTIP and beyond - the path for reform Enhancing the right to regulate and moving from current ad hoc arbitration towards an Investment Court», Comisión Europea, acceso el 12 de mayo de 2017, http://trade.ec.europa.eu/doclib/docs/2015/may/ tradoc_153408.PDF.

24 «State of the Union - A Multilateral Investment Court», Comisión Europea, acceso el 24 de abril de 2018, http://rade.ec.europa.eu/doclib/docs/2017/september/tradoc_156042.pdf. 
Finalmente, el 18 de abril del 2018, se publicaron dos nuevas versiones del borrador del ALCUES del 2015: el APIUES para el ámbito de las inversiones y el arbitraje, materias de competencia compartida entre la Unión y los Estados Miembros, y el ALCUES para el resto de materias competencia exclusiva de la Unión, siguiendo la diferenciación establecida por el Dictamen $2 / 15$. Con esta división del acuerdo se pretendía garantizar una swift EU-only firma y conclusión de aquellas partes sujetas a competencia exclusiva de la Unión ${ }^{25}$. A fecha de mayo del mismo año, la CE ha presentado el borrador de ambos acuerdos al Consejo de Ministros para su aprobación. Obtenida esta, será necesaria una segunda aprobación del PE para que el ALCUES y el APIUES del 2018 puedan entrar en vigor ${ }^{26}$.

Dentro del borrador del APIUES del 2018, el Capítulo III sobre resolución de conflictos no menciona en ningún momento el STI cuya incorporación se había prometido en diciembre del 2017. Por ello, resulta esencial realizar un análisis de fondo para determinar de forma efectiva si los borradores del ALCUES del 2015 y del APIUES del 2018 reflejan una evolución del arbitraje de diferencias Estado-inversor a un STI. Comenzamos así con un estudio del régimen de arbitraje previsto en el ALCUES del 2015.

\section{EL RÉGIMEN DE ARBITRAJE DE INVERSIONES EN EL ACUERDO DE LIBRE COMERCIO ENTRE LA UNIÓN EUROPEA Y SINGAPUR DEL 2015}

\section{Terminación de los Acuerdos sobre Promoción y Protección Recíproca de las Inversiones y las sunset clauses}

Para analizar adecuadamente el régimen de arbitraje de inversiones en el borrador del ALCUES del 2015, debemos explicar primero que el ALCUES terminaba de forma automática los efectos de los doce APPRIs concluidos de forma bilateral por algunos de los Estados Miembros de la UE con Singapur con anterioridad al ALCUES ${ }^{27}$. Concretamente, el ALCUES terminaba los efectos de los APPRIs y los sustituía una vez entrado el Acuerdo en vigor (art. 9.10.1 ALCUES) ${ }^{28}$.

${ }^{25}$ David Kleimann, «Beyond the Shadow of the Veto: Economic Treaty-Making inthe European Union after Opinion 2/15», en Institutionalisation Beyond the Nation State, ed. por Élaine Fahey, Series Studies in European Economic Law and Regulation, Vol. 10 (Cham: Springer, 2018), edición en PDF, 20.

26 «Next steps towards finalising the EU-Singapore agreements», Comisión Europea, acceso el 22 de abril de 2018, http://trade.ec.europa.eu/doclib/docs/2018/april/tradoc_156716.pdf.

${ }_{27}$ Elms, «Understanding the EU-Singapore Free Trade Agreement», 53.

28 Acuerdo de mayo del 2015 de Libre Comercio entre la Unión Europea y Singapur. Borrador. Cap. IX. 
Según Nikos Lavranos, al tener la UE competencia exclusiva en materia de inversiones (ver epígrafe V.1), la Unión podía terminar los efectos de los APPRIs mediante el ALCUES aunque no fuese parte de los mismos. En el supuesto análogo del AECG, decía Francisco Pascual Vives que la terminación de los APPRIs era necesaria para evitar una «situación de asimetría jurídica entre los Estados miembros de la UE que han celebrado APPRIs con Canadá y los Estados Unidos de América y el resto de Estados miembros» y, en último lugar, «garantizar·el afán armonizador que parece guiar la actividad de la Comisión Europea ${ }^{29}$. Sin embargo, otra parte de la doctrina se posicionaba en contra de la terminación de los APPRIs concluidos con Singapur, argumentando que iba en contra del artículo 59 del Convenio de Viena sobre el Derecho de los Tratados de 1969 (CVDT, ver epígrafe V.1) ${ }^{30}$.

El ALCUES terminaba no sólo los APPRIs previos, sino también las conocidas como sunset clauses contenidas en los mismos; i.e. aquellas clausulas que mantienen «sus efectos por un determinado periodo de tiempo tras su terminación, con el propósito de conferir una protección adicional a las inversiones extranjeras establecidas durante su vigencia» ${ }^{31}$. La terminación de las sunset clauses de APPRIs anteriores venía establecida en el artículo 9.10.2 ALCUES, que decía así32:

«En el caso de la aplicación provisional prevista en el párrafo 4 del artículo 17.12 [Entrada en Vigor] de este Acuerdo, incluyendo este Capítulo, la aplicación de las provisiones de los acuerdos listados en el Anexo 9-D, así como los derechos y obligaciones de ahí derivados, deberá suspenderse a fecha de la aplicación provisional. En caso de terminación de la aplicación provisional de este Acuerdo, la suspensión deberá cesar y los acuerdos listados en el Anexo 9-D deberán tener efecto».

Tradicionalmente, las sunset clauses suelen proteger a los inversores durante períodos de 10 a 20 años, pero en el caso del ALCUES la terminación repentina de las sunset clauses implicaba que se perdía esa garantía jurídica ${ }^{33}$. Para paliar la consecuente falta de seguridad jurídica, el ALCUES permitía excepcionalmente a los inversores recurrir al arbitraje de inversiones de los

${ }^{29}$ Francisco Pascual Vives, «El futuro del arbitraje de inversión en los acuerdos internacionales celebrados por la Unión Europea», en Revista Electrónica de Estudios Internacionales, n. 33 (2017), edición en PDF, 31.

${ }^{30}$ Nikos Lavranos, «In-depth briefing: Mixed exclusivity - The Court of Justice of the EU's Opinion on the EU Singapore FTA», en Borderlex (2017), edición en PDF, 4.

31 Pascual Vives, «El futuro del arbitraje de inversión en los acuerdos internacionales celebrados por la Unión Europea», 30-31.

${ }^{32}$ Pascual Vives, «El futuro del arbitraje de inversión en los acuerdos internacionales celebrados por la Unión Europea», 30. Traducción de la autora.

${ }^{33}$ Lavranos, «In-depth briefing: Mixed exclusivity - The Court of Justice of the EU's Opinion on the EU Singapore FTA», 5. 
APPRIs anteriores durante un período de tres años desde la entrada en vigor del Acuerdo y solamente para disputas surgidas durante el período de vigencia de los APPRIs (art. 9.10.3 ALCUES).

\section{Remisión a reglas internacionales}

El borrador del ALCUES del 2015 adoptaba un régimen de arbitraje de diferencias Estado-inversor muy innovador, basado en las reglas de dos sistemas de arbitraje internacional fundamentales. En primer lugar, se inspiraba de las reglas del CIADI para regular las siguientes áreas:

1. Previsión de la imputación de costes y remuneración de los árbitros. De acuerdo con la CE, el borrador del ALCUES del 2015 y el AECG eran únicos en su tipo, pues abarcaban un ámbito de regulación inusual en los acuerdos de inversión: la imputación de costes y la consecuente regulación de la remuneración de los árbitros ${ }^{34}$. En efecto, establecía el borrador del ALCUES que «los costes del arbitraje (incluyendo tarifas y gastos de los árbitros) así como otros costes razonables (incluyendo costes de representación legal y asistencia) deberán soportarse por la parte perdedora (principio del «perdedor lo paga todo») $)^{35}$ y que estos costes se regirían en particular por lo dispuesto en el artículo 14.1 de las Regulaciones Administrativas y Financieras de la Convención del CIADI (art. 9.26 ALCUES); y,

2. Mayor flexibilidad en el nombramiento de árbitros. Por regla general, los árbitros en mecanismos de resolución de diferencias Estado-inversor eran elegidos necesariamente por las partes ${ }^{36}$. Sin embargo, en el borrador del ALCUES los árbitros eran elegidos por las partes $\mathrm{y}$, a falta de elección de las partes, por un Comité de Comercio de una lista de 15 árbitros preestablecida por los Estados que fuesen partes del procedimiento (art. 9.18.3 ALCUES) ${ }^{37}$.

${ }^{34}$ Steffen Hindelang y Teoman M. Hagemeyer, In Pursuit of an International Investment Court: Recently negotiated investment chapters in EU Comprehensive FTA in comparative perspective (Parlamento Europeo, Directorate-General for External Policies, 2017), edición en PDF, 155.

${ }^{35}$ Hindelang y Hagemeyer, In Pursuit of an International Investment Court: Recently negotiated investment chapters in EU Comprehensive FTA in comparative perspective, 154-155, traducción de la autora.

${ }^{36}$ Hindelang y Sassenrath, The investment chapters of the EU's international trade and investment agreements in a comparative perspective, 10 y 88-93.

${ }^{37}$ No consta información sobre la composición y organización de este Comité en el borrador del ALCUES del 2015 ni en documento complementario de la UE (acceso el 22 de marzo de 2018). 
Segundo, el ALCUES hacía referencia a las reglas de la CNUDMI en cuanto $\mathrm{a}^{38}$ :

1. Establecer un código de conducta para los árbitros. El Anexo 9-F del borrador del ALCUES regulaba exclusivamente el comportamiento de los árbitros y concretamente las obligaciones de declaración de aquellos asuntos que pudiesen comprometer su decisión, obligaciones generales, independencia e imparcialidad, confidencialidad, y costes. En particular, el Anexo 9-F brindaba la posibilidad a los Estados partes del procedimiento de examinar la correcta conducta de los árbitros, en contra de la tendencia general por la que asociaciones profesionales o instituciones de arbitraje asumían esa responsabilidad ${ }^{39}$;

2. Garantizar la transparencia del procedimiento arbitral. El Anexo 9-G del borrador del ALCUES se inspiraba directamente del artículo 3.1 de las Reglas de Transparencia del 2014 de la CNUDMI y permitía al público acceder a ciertos documentos, entre los cuales: las consultas, la notificación de la intención de recurrir al arbitraje, la presentación de una reclamación de arbitraje, y documentos judiciales (art. 1.1 Anexo 9-G). Tomando como base los artículos 3.3 y 7 de las Reglas de Transparencia, el Anexo 9-G permitía además al público acceder a documentos distintos de los anteriores previa autorización del tribunal arbitral, salvo que ello comprometiese información confidencial (arts. 1.2 y 4 Anexo 9-G) ${ }^{40}$. Esta preocupación por una mayor transparencia recordaba a la Declaración de Laeken del 2001 por la que el CE prometía «mejorar la gobernanza de la UE mediante más democracia, transparencia y eficiencia ${ }^{41} ; \mathrm{y}$,

3. Garantizar el acceso público a las audiencias de las disputas sobre inversiones. Tomando como base el artículo 6 de las Reglas de Transparencia, el Anexo 9-G hacía las audiencias públicas salvo que una parte solicitase lo contrario con el fin de salvaguardar información confidencial (art. 2 Anexo 9-G) ${ }^{42}$.

${ }^{38}$ Acuerdo de mayo del 2015 de Libre Comercio entre la Unión Europea y Singapur. Borrador. Anexos 9-F y 9-G.

${ }^{39}$ Hindelang y Sassenrath, The investment chapters of the EU's international trade and investment agreements in a comparative perspective, 10.

${ }^{40}$ Hindelang y Hagemeyer, In Pursuit of an International Investment Court: Recently negotiated investment chapters in EU Comprehensive FTA in comparative perspective, 121.

${ }^{41}$ Luis Enrique De La Villa Gil, «El futuro de la Unión Europea. Declaración de Laeken», en Revista del Ministerio de Trabajo y Asuntos Sociales, n. 37 (2001), edición en PDF, 226.

${ }^{42}$ Hindelang y Hagemeyer, In Pursuit of an International Investment Court: Recently negotiated investment chapters in EU Comprehensive FTA in comparative perspective, 122. 


\section{Regulación de las consultas}

Con el objetivo de hacer los procedimientos de arbitraje más efectivos, el borrador del ALCUES del 2015 «institucionalizaba» la fase de las consultas y construía todo el procedimiento de arbitraje de diferencias Estado-inversor en torno a las mismas.

Concretamente, el borrador del ALCUES comenzaba otorgando al demandante un plazo para iniciar las consultas de tres meses desde que tuvo conocimiento del daño o de un año desde que cesó de recurrir a los tribunales nacionales y entendía iniciadas esas consultas con la mera solicitud de las mismas (arts. 9.13.1 y 3 ALCUES). Iniciadas las consultas, el demandante podía entregar al demandado la notificación de la intención de someter una disputa a arbitraje o requerir la identificación del demandado en un plazo de tres meses (art. 9.15.1 ALCUES), salvo que la reclamación de identificación del demandado fuese enviada a la UE, en cuyo caso el plazo se reducía a dos meses (art. 9.15.2 ALCUES). Notificado el demandado de la intención de someter una disputa a arbitraje, el demandante disponía de otros tres meses para presentar la demanda ante un foro de arbitraje (art. 9.16.1 ALCUES). Vemos así como se construía un procedimiento dividido en tres fases, en el que cada una dependía de la consecución de la anterior y las consultas formaban la base misma del proceso.

Según el PE, la clara estructura del procedimiento garantizaba cierta seguridad jurídica a las partes, pues limitaba el objeto de las disputas antes incluso de llegar al arbitraje. No obstante, también podía generar problemas de abuso del derecho y precisamente para evitar esta situación el borrador del ALCUES incorporó la siguiente excepción a los plazos del artículo 9.13.3 explicados anteriormente: a falta de consultas del demandante en los plazos indicados, podía éste presentar la demanda de arbitraje siempre y cuando las causas del retraso fuesen imputables al demandado (art. 9.13.5 ALCUES) ${ }^{43}$.

\section{Adopción del no U-turn}

Tradicionalmente, cuando un inversor quiere dirigir una demanda contra un Estado tiene que elegir si dirigirla por la vía judicial nacional o si recurrir al arbitraje internacional, y una vez iniciado el procedimiento judicial o arbitral, según el caso, no puede cambiarse a la otra vía. Este mecanismo es el conocido como «fork in the road o bifurcación en la carretera» ${ }^{44}$.

${ }^{43}$ Hindelang y Hagemeyer, In Pursuit of an International Investment Court: Recently negotiated investment chapters in EU Comprehensive FTA in comparative perspective, 37.

${ }_{44}$ Pascual Vives, «El futuro del arbitraje de inversión en los acuerdos internacionales celebrados por la Unión Europea», 25. 
Por el contrario, el borrador del ALCUES del 2015 ofrecía al inversor la posibilidad de elegir si iniciar el procedimiento en vía judicial nacional o si recurrir al arbitraje internacional, pero con posibilidad en caso de comenzar el procedimiento en tribunales nacionales de dirigirse luego a la vía arbitral «si considerara que su reclamación no está siendo debidamente atendida» ${ }^{45}$. Esta última estrategia, conocida como «no U-turn» (prohibido dar la vuelta), tenía por objetivo promover la resolución de conflictos sobre inversiones por la vía judicial naciona ${ }^{46}$ a la vez que facilitaba el acceso del inversor al arbitraje de diferencias Estado-inversor. Ahora bien, el cambio al procedimiento arbitral era irreversible y el inversor no podía volver a posteriori a la vía judicial $^{47}$. A estos efectos y para evitar procedimientos paralelos, el inversor demandante debía retirar cualquier reclamación pendiente en los tribunales nacionales y declarar que no presentaría ninguna demanda con el mismo objeto antes de haber tomado el tribunal arbitral su decisión final (art. 9.17.1.f) ALCUES).

Así definidas las principales características del arbitraje de diferencias Estado-inversor del borrador del ALCUES del 2015, analizaremos ahora el régimen de arbitraje de inversiones del APIUES del 2018.

\section{EL RÉGIMEN DE ARBITRAJE DE INVERSIONES EN EL ACUERDO DE PROTECCIÓN DE LAS INVERSIONES ENTRE LA UNIÓN EUROPEA Y SINGAPUR DEL 2018}

\section{Indicios de un Sistema de Tribunales de Inversiones}

El STI es un régimen de arbitraje de inversiones incorporado en dos ALCs muy importantes de la UE: a) la ATCI, aún bajo negociación y con el potencial de convertirse en el mayor acuerdo comercial del mundo según la $\mathrm{CE}^{48}$; y, b) el AECG, bajo aplicación provisional y «el primer acuerdo firmado por la UE que incluye clausulas para la protección de las inversiones y

${ }^{45}$ Cortes Generales, «Diario de Sesiones del Congreso de los Diputados», acceso el 21 de abril de 2018, http://www.congreso.es/public_oficiales/L10/CONG/DS/CO/DSCD10-CO-832.PDF.

46 Pascual Vives, «El futuro del arbitraje de inversión en los acuerdos internacionales celebrados por la Unión Europea», 25.

${ }^{47}$ Hindelang y Hagemeyer, In Pursuit of an International Investment Court: Recently negotiated investment chapters in EU Comprehensive FTA in comparative perspective, 79 .

${ }^{48}$ George K. Zestos y Christopher W. Coffman, «Transatlic Economic Integration», en The TTIP - The Transatlantic Trade and Investment Partnership between the European Union and the United States, ed. por Joaquín Roy y Roberto Domínguez (Miami: Miami-Florida European Union Center of Excellence, 2014), 73. 
un mecanismo de resolución de conflictos para aplicar esos derechos de protección $\rangle^{49}$.

Comenzando por la ATCI y a fecha de mayo del 2018, sabemos que el STI estará formado por «un Tribunal de Primera Instancia (Tribunal de Inversiones), compuesto de 15 jueces, y un Tribunal de Apelaciones, compuesto de 6 jueces, cuya cualificación técnico-jurídica sería comparable a la de los jueces de la Corte Internacional de Justicia o los miembros del Órgano de Apelación de la $\mathrm{OMC} \gg{ }^{50}$. Los miembros de los Tribunales no serán elegidos por los inversores como ocurría en el arbitraje de diferencias Estadoinversor y ésta es una diferencia esencial con el sistema anterior ${ }^{51}$.

De forma general, crear un STI implica además que ${ }^{52}$ :

«- se crearía un sistema público de tribunales de inversiones, formado por un Tribunal de Primera Instancia y un Tribunal de Apelación;

- las resoluciones serían adoptadas por jueces designados por autoridades públicas y que tendrían unas elevadas cualificaciones, comparables a las exigidas a los miembros de los tribunales internacionales permanentes, como el Tribunal Internacional de Justicia y el Órgano de Apelación de la $\mathrm{OMC}$;

- el nuevo Tribunal de Apelación funcionaría con principios similares al Órgano de Apelación de la OMC;

- se definiría con precisión la capacidad de los inversores para presentar un asunto ante el Tribunal, y se limitaría a casos concretos, como los de discriminación específica por sexo, raza, religión o nacionalidad, expropiación sin indemnización o denegación de justicia;

- el derecho de los gobiernos a legislar quedaría consagrado y garantizado en las disposiciones de los acuerdos comerciales y de inversión.»

En cuanto al AECG, el STI aportará aquí también cambios a nivel del nombramiento de los miembros de los Tribunales, que ya no serán elegidos por las partes como ocurría en el arbitraje de diferencias Estado-inversor ${ }^{53}$.

${ }^{49}$ Roberto Harte y Laura Puccio, From arbitration to the investment court system (ICS) - The evolution of CETA rules (Servicios De Estudios Del Parlamento Europeo (EPRS), 2017), edición en PDF, 4.

${ }^{50}$ Antonio Segura Serrano, «El Acuerdo de Libre Comercio entre la UE y Canadá (CETA): Una evaluación de la política comercial de la UE», Revista Electrónica de Estudios Internacionales 30 (2015): 16-17, doi:10.17103/reei.33.02.

${ }_{51}$ Harte y Puccio, From arbitration to the investment court system (ICS) - The evolution of CETA rules, 7.

52 «La Comisión propone un nuevo Sistema de Tribunales de Inversiones para la ATCI y para otras negociaciones comerciales y de inversión de la UE», Comisión Europea, acceso el 25 de abril de 2018, http://europa.eu/rapid/press-release_IP-15-5651_es.htm.

${ }_{53}$ Harte y Puccio, From arbitration to the investment court system (ICS) - The evolution of CETA rules, 5. 
Además de esta medida, se ha previsto hacer un STI que «será público; no se basará en tribunales temporales; tendrá jueces y profesionales independientes designados por la UE y Canadá, y sujetos a las más rigurosas normas éticas a través de un estricto código de conducta; trabajará con transparencia, pues celebrará públicamente sus audiencias y publicará los documentos presentados durante la tramitación de los casos» ${ }^{54}$.

Analizar la configuración del STI en la ATCI y en el AECG nos permite entender mejor en qué consiste el STI y cómo se refleja en otros ALCs de la Unión, en particular en el APIUES. En efecto, un estudio exhaustivo revela que el APIUES comparte con la ATCI y el AECG las siguientes características ${ }^{55}$ :

1. Composición. El borrador del APIUES prevé el establecimiento de un Tribunal de Primera Instancia (o Tribunal de Inversiones) y de un Tribunal de Apelaciones. Los jueces de ambos órganos serían elegidos de la siguiente manera: dos por la UE, dos por Singapur y dos por ambas partes conjuntamente (arts. 3.9.2 y 3.10.2 APIUES). Además, estos jueces deberían tener suficiente cualificación técnico-jurídica como para ser jueces titulares en sus países respectivos (arts. 3.9.4 y 3.10.4 APIUES).

2. Naturaleza. Las instituciones del STI deberán tener carácter internacional y permanente, aunque se limita esta medida al Tribunal de Apelaciones (art. 3.10.1 APIUES).

3. Remuneración. Los honorarios de los jueces (incluyendo tarifas y gastos) de ambos Tribunales podrán tener carácter permanente siempre y cuando: a) así lo establezca un Comité de Comercio que se reunirá cada dos años y que estará compuesto por representantes de la UE y de Singapur, entre los cuales el Ministro de Comercio e Industria de Singapur y el responsable de la CE sobre Comercio; b) trabajen en el STI a jornada completa, y; c) trabajen en el STI de forma exclusiva, salvo autorización del Presidente del Tribunal que permita lo contrario (arts. 3.9.15, 3.10.13 y 4.1.1 y 2 APIUES).

4. Ética. Los jueces deberán «ser elegidos de entre personas cuya independencia esté garantizada» para evitar cualquier tipo de conflicto de intereses. Si considerasen que el juez en cuestión incurre efectivamente en un conflicto de intereses, las partes podrán recusar la elección del juez ante el Presidente del Tribunal relevante (art. 3.11.1 y 2 APIUES).

${ }^{54}$ «El AECG explicado», Comisión Europea, acceso el 13 de abril de 2018, http:// ec.europa.eu/trade/policy/in-focus/ceta/ceta-explained/index_es.htm.

55 Acuerdo de abril del 2018 de Protección de las Inversiones entre la Unión Europea y Singapur. Borrador. 
5. Transparencia. Los procedimientos arbitrales del STI deberán regirse por el principio de transparencia, con audiencias públicas y documentos accesibles al público (art. 3.16 APIUES).

6. Cumplimiento efectivo. Siguiendo el principio de la buena fe, las partes deberán adoptar las medidas necesarias para cumplir con lo establecido por la sentencia arbitral del Tribunal respectivo (art. 3.33 APIUES).

\section{Sobre las disposiciones derivadas de reglas internacionales}

Al igual que el borrador del Capítulo IX del ALCUES del 2015, el borrador del Capítulo III del APIUES del 2018 se organiza en torno a las reglas del CIADI y de la CNUDMI, pero introduce ciertas modificaciones.

En primer lugar, el APIUES se distancia del ALCUES en cuanto a las siguientes reglas, inspiradas en un principio del CIADI:

1. Previsión de la imputación de costes y remuneración de los árbitros. El APIUES retoma lo establecido por el artículo 9.26 del borrador del ALCUES, imputando los costes del arbitraje a la parte perdedora (art. 3.21.1 APIUES) y remitiéndose al artículo 14.1 de las Regulaciones Administrativas y Financieras de la Convención del CIADI (Art. 3.9.14 APIUES), pero introduce la siguiente modificación: los honorarios del Presidente o Vice-Presidente del Tribunal correspondiente (incluyendo tarifas y gastos) deberán abonarse por ambas partes y por partes iguales a una cuenta específica supervisada por la Secretaría del CIADI (art. 3.9.13 APIUES); y,

2. Mayor claridad en el nombramiento de árbitros. Mientras el borrador del ALCUES del 2015 asignaba el nombramiento de los árbitros a las partes o, en su defecto, a un Comité de Comercio, el borrador del APIUES atribuye esta facultad a las partes exclusivamente, siguiendo el formato explicado anteriormente (ver epígrafe IV.1).

En segundo lugar, el borrador del APIUES modifica lo establecido por el ALCUES con respecto de las reglas de la CNUDMI en lo siguiente:

1. Mayor regulación del código de conducta para los árbitros. Al igual que el Anexo 9-F del borrador del ALCUES, el borrador del Anexo 7 del APIUES regula exclusivamente el comportamiento de los árbitros y en particular las obligaciones de declaración de aquellos asuntos que puedan comprometer su decisión, obligaciones generales, independencia e imparcialidad, confidencialidad, y costes. Sin embargo, en este Anexo 7 puede apreciarse una mayor regulación en cuanto a las 
obligaciones de los antiguos jueces del Tribunal, que no podrán relacionarse con asuntos sobre inversiones relacionados ni con las partes durante un plazo de tres años (apartados 16 y 17 Anexo 7 APIUES). Además, se añade un apartado relativo a la creación de un Comité Consultivo, que asistirá al Presidente del Tribunal respectivo en su trabajo y que estará compuesto por el Vice-Presidente y el juez de mayor edad de dicho Tribunal (apartados 24 Anexo 7 APIUES); y,

2. Menor transparencia en el procedimiento arbitral. Al igual que el borrador del Anexo 9-G del ALCUES, el borrador del Anexo 8 del APIUES se inspira directamente del artículo 3.1 de las Reglas de Transparencia del 2014 de la CNUDMI pues contempla el acceso público a las consultas, la notificación de la intención de recurrir al arbitraje, la presentación de una reclamación de arbitraje (ahora denominada presentación de una reclamación ante el Tribunal) y otros documentos judiciales. Sin embargo, el APIUES introduce ciertas modificaciones respecto del borrador del Anexo 9-G del ALCUES y en particular limita la transparencia del proceso arbitral, en tanto que lista de forma exhaustiva todas aquellas situaciones en las que el público no podrá acceder a información confidencial e, incluso cuando exceptúa de esta regla situaciones excepcionales y/o donde la información es necesaria para el correcto funcionamiento del proceso arbitral, permite limitar o retrasar la publicación de esa información (art. 4 Anexo 8 APIUES).

\section{Sobre la regulación de las consultas}

El borrador del APIUES mantiene la estructura en tres fases del procedimiento de arbitraje previsto en el borrador del ALCUES del 2015, pero extiende la duración de muchos de los plazos iniciales (ver epígrafe III.3).

En efecto, el demandante tiene en esta nueva versión un plazo para iniciar las consultas de treinta meses desde que tuvo conocimiento del daño o de un año desde que cesó de recurrir a los tribunales nacionales (art. 3.3.3 APIUES). Iniciadas las consultas, el demandante puede entregar al demandado la notificación de la intención de someter una disputa a arbitraje o requerir la identificación del demandado en un plazo de dieciocho meses (art. 3.3.4 APIUES). El ALCUES exceptúa de esta regla el supuesto en que se envíe la reclamación de la identidad del demandado a la UE, donde el plazo se reduce a dos años, pero el APIUES tan sólo menciona la posibilidad de realizar tal acción sin decir nada sobre el tiempo (art. 3.3.6 APIUES). En último lugar, no se cambia el plazo de tres meses que tiene el demandante para presentar la demanda ante un foro de arbitraje una vez se haya notificado al demandado de la intención de someter una disputa a arbitraje (art. 3.6.1 APIUES). 
Con el objetivo de garantizar un mínimo de seguridad jurídica, se mantiene también la regla del borrador del ALCUES por la que, a falta de consultas del demandante en los plazos de los apartados 3 y 4, podrá éste presentar la demanda de arbitraje siempre y cuando las causas de dicho retraso sean imputables al demandado y que el demandante actúe lo antes posible (art. 3.3.5 APIUES).

\section{Sobre la adopción del no U-turn}

Al igual que el borrador del ALCUES, el borrador del APIUES ofrece al inversor la posibilidad de elegir iniciar el procedimiento en vía judicial nacional y dirigirse luego a la vía arbitral, y este cambio es irreversible.

Sin embargo, el APIUES introduce ciertos matices: a) el inversor demandante deberá retirar cualquier reclamación pendiente, no sólo en los tribunales nacionales como ocurría en el borrador del ALCUES, sino que también en los tribunales naciones o internacionales bajo derecho nacional o internacional; b) el inversor demandante deberá declarar que no presentará ninguna demanda con el mismo objeto, no sólo hasta que el tribunal arbitral haya tomado su decisión final como ocurría en el borrador del ALCUES, sino que la obligación se extenderá a futuro; y, c) el inversor demandante deberá, añade el APIUES, declarar que «no ejecutará ninguna sentencia arbitral hasta que sea definitiva y no intentará apelar, recurrir, anular, revisar o iniciar procedimientos similares ante una corte o tribunal internacional o nacional» (art. 3.7.1.f) APIUES).

A partir del análisis realizado hasta el momento, vemos que el borrador del APIUES del 2018 ha cambiado el fondo de numerosas disposiciones sobre arbitraje de inversiones previstas en el antiguo ALCUES del 2015. Sin embargo, el análisis se ha limitado a estudiar el contenido de ambos acuerdos a partir de los propios acuerdos y la doctrina, sin mencionar en ningún momento la jurisprudencia existente al respecto. Por lo tanto, analizaremos ahora los problemas legales planteados por el ALCUES/APIUES desde un punto de vista jurisprudencial.

\section{OBSTÁCULOS LEGALES AL ACUERDO DE LIBRE COMERCIO ENTRE LA UNIÓN EUROPEA Y SINGAPUR}

\section{El artículo 59 del Convenio de Viena sobre el Derecho de los Tratados y el Dictamen 2/15}

Como adelantábamos anteriormente (ver epígrafe II.1), el artículo 59 CVDT fue una de las principales críticas alzadas por la doctrina contra el efecto de sustitución de los APPRI que tenía el borrador del ALCUES del 
2015. Concretamente, el debate se formaba en torno a la siguiente disposición del artículo 59.156:

«1. Se considerará que un tratado ha terminado si todas las partes en él celebran ulteriormente un tratado sobre la misma materia y:

a) se desprende del tratado posterior o consta de otro modo que ha sido intención de las partes que la materia se rija por ese tratado; o

b) las disposiciones del tratado posterior son hasta tal punto incompatibles con las del tratado anterior que los dos tratados no pueden aplicarse simultáneamente.»

La problemática radicaba en quiénes eran las partes del borrador del ALCUES del 2015. En los APPRIs, las partes del lado europeo eran claramente los Estados Miembros implicados, pero quién intervenía en el ALCUES: ¿la UE sola o la UE con los Estados Miembros? Es decir, ¿nos encontrábamos ante un acuerdo exclusivo o un acuerdo mixto?

Si considerábamos que tanto la UE como los Estados Miembros intervenían en el ALCUES, éste era perfectamente compatible con el artículo 59 CVDT. Para ilustrar esta afirmación, Nikos Lavranos recuerda el caso del GATT en 1947, firmado por los Estados Miembros y éstos luego sustituidos por la Comunidad Económica Europea $(\mathrm{CEE})^{57}$. En cambio, si considerábamos que la UE intervenía sola, permitir la terminación de los APPRIs mediante el ALCUES vulneraba de forma directa el artículo 59 CVDT. La Abogado General Eleanor Sharpston decía al respecto en las conclusiones presentadas el 21 de diciembre de 2016 que $^{58}$ :

«En estas circunstancias, no creo que el Derecho internacional, en su estado actual, permita concluir que la Unión puede subrogarse de forma automática en un acuerdo internacional celebrado por los Estados miembros del que no es parte y posteriormente darlo por terminado. Esa norma

${ }^{56}$ Convenio de Viena, de 23 de mayo de 1969, de las Nacionales Unidas sobre el Derecho de los Tratados (BOE n. ${ }^{\circ} 142$ de 13 de junio de 1980).

${ }^{\mathrm{C}}$ onviene señalar que el artículo 59.1 CVDT coincide con el artículo 59.1 de la Convención de Viena sobre el Derecho de los Tratados celebrados entre Estados y Organizaciones Internacionales (CVDTEOI) de 1986, pero que sólo la CVDT planteó dudas sobre el ALCUES, puesto que: a) las disposiciones de la CVDTEOI sólo son aplicables a partir de la entrada en vigor de la Convención (art. 4 CVDTEOI); y b) la entrada en vigor precisa de la ratificación de la Convención por un mínimo de 35 Estados (art. 85.1 CVDTEOI), condición ésta que todavía no se ha producido (acceso el 21 de abril de 2018).

${ }^{57}$ Lavranos, «In-depth briefing: Mixed exclusivity - The Court of Justice of the EU's Opinion on the EU Singapore FTA», 4, traducción de la autora.

${ }_{58}$ Conclusiones de la Abogado General Sra. Eleanor Sharpston, de 21 de diciembre de 2016, C-2/15, EU:C:2016:992, 396. 
constituiría una excepción al principio fundamental del consentimiento en la instauración de normas internacionales.»

La CE defendía la competencia exclusiva de la UE mientras que los Estados Miembros y el Consejo de la UE se posicionaban en favor de un acuerdo mixto. Para resolver este debate, la CE pidió el marzo del 2015 al TJUE que se pronunciara sobre el reparto competencial y la naturaleza del ALCUES. El Pleno del TJUE respondió a esta petición en el Dictamen 2/15, de 16 de mayo del 2017, en el que declaró que el ALCUES estaba incluido en la competencia exclusiva de la Unión, excepto en tres áreas: a) la Sección A del Capítulo IX sobre protección de las inversiones; b) la Sección B del Capítulo IX sobre el arbitraje de diferencias Estado-inversor; y, c) «las disposiciones de los Capítulos 1 (Objetivos y definiciones generales), 14 (Transparencia), 15 (Solución de diferencias entre las Partes), 16 (Mecanismo de mediación) y 17 (Disposiciones institucionales, generales y finales) del Acuerdo, en la medida en que se refieran a las disposiciones del mencionado capítulo 9 y en tanto en cuanto estas últimas se incluyan en el ámbito de una competencia compartida entre la Unión y los Estados miembros $\rangle^{59}$. Esta decisión es legalmente vinculante para los Estados Miembros, «salvo modificación de éste o revisión de los Tratados» (art. 218.11 TFUE).

Empezando por la Sección A del Capítulo IX, debemos distinguir entre las IEDs y las inversiones no-directas para entender la argumentación del TJUE. Respecto de las primeras, decía el Tribunal de Luxemburgo que ${ }^{60}$ :

«... los compromisos contenidos en la sección A del capítulo 9 del Acuerdo proyectado en relación con la protección de las inversiones están incluidos en el ámbito de la política comercial común de la Unión y, por tanto, en el ámbito de la competencia exclusiva de ésta con arreglo al artículo 3 TFUE, apartado 1, letra e), en la medida en que se refieran a las «inversiones extranjeras directas» en el sentido del artículo 207 TFUE, apartado 1, entre la Unión y la República de Singapur».

Sin embargo, las inversiones extranjeras no-directas se entendían competencia compartida en base de los siguientes argumentos:

1. La celebración por la UE de un acuerdo internacional sobre inversiones extranjeras no-directas no estaba ««prevista en un acto legislativo de la Unión» en el sentido del citado artículo 3, apartado $2 \gg^{61}$, pero a la vez era necesaria la participación de la UE para alcanzar los

${ }^{59}$ Cremona, «Shaping EU Trade Policy post-Lisbon: Opinion 2/15 of 16 May 2017», 231-232, traducción de la autora.

${ }^{60}$ Dictamen del Pleno del TJUE, de 16 de mayo de 2017, C-2/15, EU:C:2017:376, 225.

${ }^{61}$ Dictamen del Pleno del TJUE, de 16 de mayo de 2017, C-2/15, EU:C:2017:376, 236. 
objetivos de los tratados siguiendo lo establecido por el art 216.1 TFUE. En particular, se buscaba alcanzar el objetivo de libre circulación de capitales y de pagos entre los Estados miembros y los terceros Estados previsto en el art. 63 TFUE e incluido en la competencia compartida de la UE y los Estados Miembros del art. 4.2.a) TFUE ${ }^{62}$; y,

2. La competencia de la Unión en relación con el art. 216.1 TFUE era «también de naturaleza compartida, ya que el artículo 4 TFUE, apartado 1, establece que la Unión «dispondrá de competencia compartida con los Estados miembros cuando los Tratados le atribuyan una competencia que no corresponda a los ámbitos mencionados en los artículos 3 y $6 »$, lo que ocurre en este caso» ${ }^{63}$, y las inversiones extranjeras no-directas quedan fuera de la política comercial común del artículo 3.1.e) TFUE y dentro del mercado interior y de la política social del artículo 4.1 y $2 \mathrm{TFUE}^{64}$.

En cuanto a la Sección B, regulaba ésta el arbitraje de diferencias Estadoinversor y permitía al inversor-demandante someterse a los tribunales de Singapur o de un Estado Miembro de la UE (art. 9.17 ALCUES). Según el TJUE, «un régimen de esta índole, que sustrae diferencias a la competencia jurisdiccional de los Estados miembros, no puede tener carácter meramente auxiliar en el sentido de la jurisprudencia recordada en el apartado 276 del presente dictamen, $\mathrm{y}$, en consecuencia, no puede establecerse sin el consentimiento de éstos» ${ }^{65}$; es decir que era necesaria la competencia compartida entre la Unión y los Estados Miembros también para esta segunda Sección del Capítulo IX.

Como explicábamos anteriormente (ver epígrafe II), el Dictamen 2/15 del TJUE ha sido muy importante en la configuración del APIUES tal y como lo conocemos a fecha de mayo del 2018. En efecto, la división del borrador del ALCUES del 2015 en dos acuerdos nuevos se ha realizado siguiendo el reparto competencial del TJUE, con las competencias compartidas sobre inversiones pasando a ser reguladas por el APIUES y el resto de competencias exclusivas siendo reguladas por el nuevo ALCUES del 2018. En sentido contrario, calcular los efectos de la Sentencia del TJUE en el caso Achmea que explicaremos ahora sobre el APIUES resulta más difícil.

${ }^{62}$ Dictamen del Pleno del TJUE, de 16 de mayo de 2017, C-2/15, EU:C:2017:376, 239-241.

${ }_{63}$ Dictamen del Pleno del TJUE, de 16 de mayo de 2017, C-2/15, EU:C:2017:376, 242.

${ }^{64}$ Dictamen del Pleno del TJUE, de 16 de mayo de 2017, C-2/15, EU:C:2017:376, 242-243.

${ }^{65}$ Dictamen del Pleno del TJUE, de 16 de mayo de 2017, C-2/15, EU:C:2017:376, 289-293. 


\section{La Sentencia de la Gran Sala del Tribunal de Justicia de la UE, de 6 de marzo de 2018, sobre el caso Achmea}

En el Dictamen 2/15, el TJUE analizó el problema del reparto competencial de las áreas del borrador del ALCUES del 2015 entre la UE y los Estados Miembros, pero no se pronunció sobre la compatibilidad del arbitraje de diferencias Estado-inversor con el DUE ${ }^{66}$. Esta cuestión sólo se abordó a comienzos de este año con la Sentencia de la Gran Sala del TJUE, de 6 de marzo de 2018, sobre el caso Achmea.

Para situarnos mejor, Achmea es una compañía aseguradora holandesa que comenzó un procedimiento arbitral contra la República Eslovaca en el 2008. El tribunal arbitral que aseguraba el proceso eligió Frankfurt como lugar de celebración del arbitraje ${ }^{67}$ pero Eslovaquia rechazó la jurisdicción del tribunal, argumentando que el artículo 8 APPRI Países Bajos - República Checa-Eslovaquia de 1992, en el cual se basaba la elección y jurisdicción del tribunal arbitral, era incompatible con el DUE. El tribunal arbitral rechazó este argumento y condenó a Eslovaquia a pagar a Achmea los daños y perjuicios causados. En consecuencia, Eslovaquia presentó un recurso de anulación ante el Tribunal Superior Regional de Frankfurt y, al ser rechazado, un recurso de apelación ante el Tribunal Supremo Civil y Penal de Alemania (Bundesgerichtshof), argumentando de nuevo que el arbitraje del APPRI era incompatible con el DUE ${ }^{68}$. Finalmente, el Tribunal Supremo Civil y Penal de Alemania trasladó al TJUE las siguientes dudas: a) si es incompatible la disposición sobre el arbitraje de diferencias Estado-inversor de un «APPRI interno de la Unión» (i.e. entre Estados Miembros) con el artículo 344 TFUE; b) de ser negativa la respuesta anterior, si es incompatible con el artículo 267 TFUE; c) de ser negativas las dos respuestas anteriores, si es incompatible con el artículo 18.1 TFUE ${ }^{69}$.

Comenzando por el artículo 344 TFUE, establece éste que «los Estados miembros se comprometen a no someter las controversias relativas a la interpretación o aplicación de los Tratados a un procedimiento de solución distinto de los previstos en los mismos $»^{70}$. Esta disposición esta íntimamente

${ }^{66}$ Dictamen del Pleno del TJUE, de 16 de mayo de 2017, C-2/15, EU:C:2017:376, 30.

${ }^{67}$ Siguiendo lo establecido por el artículo 20 de la Ley Modelo de la CNUDMI sobre Arbitraje Comercial Internacional de 21 de junio de 1985 con las enmiendas aprobadas en 2006 (acceso el 23 de abril de 2018).

68 Burkhard Hess, The Fate of Investment Dispute Resolution after the Achmea Decision of the European Court of Justice, n. ${ }^{\circ} 3$ (Luxemburgo: Max Planck Institute Luxembourg for Procedural Law Research Paper Series, 2018), 6.

69 Conclusiones del Abogado General Sr. Melchior Wathelet, de 19 de septiembre de 2017, Achmea, C-284/16, EU:C:2017:699, 30.

70 Tratado de Funcionamiento de la Unión Europea (TFUE), de 25 de marzo de 1957 (DOUE n. ${ }^{\circ} \mathrm{C} 202$ de 7 de junio de 2016). 
relacionada con el principio de autonomía, según el cual la UE puede concluir tratados internacionales reconociendo la jurisdicción de tribunales distintos del TJUE o los tribunales nacionales sólo si esos tribunales externos no pueden interpretar el DUE de forma definitiva y si la interpretación que hacen de las normas aplicables no afecta al «sistema de distribución de poder entre la UE y los Estados Miembros tal y como regulado en los Tratados Constitutivos, y sobre los que el TJUE tiene la máxima autoridad interpretativa ${ }^{71}$.

Respecto al artículo 344 TFUE, el Abogado General Melchior Whatelet defendió que los TBIs son compatibles con el DUE pues «los Tribunales de Inversión deberían ser considerados 'Cortes de un Estado Miembro' en el sentido del artículo 267 TFUE con el fin de permitirles enviar referencias preliminares a la Corte de Justicia Europea en relación con el impacto del Derecho de la UE sobre la interpretación de los APPRIs» ${ }^{72}$. Sin embargo, la Gran Sala del TJUE llegó a unas conclusiones drásticamente distintas en la Sentencia de 6 de marzo de 2018, defendiendo que el «carácter excepcional del tribunal arbitral» del caso Achmea impedía integrar dicho tribunal dentro del sistema jurisdiccional europeo, «ya que los Tratados no establecen ningún procedimiento judicial que permita a un inversor, como Achmea, alegar ante los tribunales de la Unión el derecho a indemnización frente a un Estado miembro que le concede un tratado bilateral de inversión como el TBI» ${ }^{73}$.

Siendo negativa la respuesta del TJUE a la primera cuestión, pasamos a analizar su respuesta a la segunda pregunta. El artículo 267 TFUE establece que:

«El Tribunal de Justicia de la Unión Europea será competente para pronunciarse, con carácter prejudicial: sobre la interpretación de los Tratados, sobre la validez e interpretación de los actos adoptados por las instituciones, órganos u organismos de la Unión. Cuando se plantee una cuestión de esta naturaleza ante un órgano jurisdiccional de uno de los Estados miembros, dicho órgano podrá pedir al Tribunal que se pronuncie sobre la misma, si estima necesaria una decisión al respecto para poder emitir su fallo. Cuando se plantee una cuestión de este tipo en un asunto pendiente ante un órgano jurisdiccional nacional, cuyas decisiones no sean susceptibles de ulterior recurso judicial de Derecho interno, dicho órgano estará obligado a someter la cuestión al Tribunal».

Aquí también, el TJUE rechazó la calificación de un tribunal arbitral de inversiones como «órgano jurisdiccional de 'uno de los Estados miembros'

${ }^{71}$ Hèctor López Bofill, «Change in the Constitutional Framework of the European Union with regard to regulating foreign direct investment», en Revista Catalana de Dret Públic, Vol. 51 (2015), edición en PDF, 19-20, traducción de la autora.

${ }^{72}$ Hess, The Fate of Investment Dispute Resolution after the Achmea Decision of the European Court of Justice, 6-7 y 10-11.

${ }^{73}$ STJUE, de 6 de marzo de 2018, Achmea, C-284/16, EU:C:2018:158, 17. 
en el sentido del artículo 267 TFUE» en base de ese carácter excepcional de los tribunales arbitrales de inversiones. Más aún, establecía el TJUE que toda disposición de un tratado concluido por algunos de los Estados Miembros únicamente «puede poner en peligro, además del principio de confianza mutua entre los Estados miembros, la preservación del carácter propio del Derecho establecido por los Tratados, garantizado por el procedimiento de remisión prejudicial previsto en el artículo 267 TFUE, por lo que no es compatible con el principio de cooperación legal» ${ }^{74}$.

Para terminar, el artículo 18.1 TFUE dice que «En el ámbito de aplicación de los Tratados, y sin perjuicio de las disposiciones particulares previstas en los mismos, se prohibirá toda discriminación por razón de la nacionalidad $\rangle^{75}$. El Tribunal de Luxemburgo no contestó a la pregunta sobre este artículo en la Sentencia, considerando que lo establecido respecto de las cuestiones anteriores constituía suficiente respuesta ${ }^{76}$.

En conclusión, el Tribunal de Luxemburgo declaró la incompatibilidad del arbitraje de diferencias Estado-inversor con los artículos 344 y 267 TFUE en aquellos APPRIs internos de la UE. Respondiendo a esta decisión, la CE ha solicitado a los Estados Miembros que «terminen los 196 APPRIs intraUE por consentimiento mutuo (art. 54 VCLT) $)^{77}$.

Terminamos así el análisis de dos referencias de la jurisprudencia esenciales para realizar un análisis comprensivo del ALCUES-APIUES y pasamos ya a las conclusiones.

\section{CONCLUSIONES}

El borrador del ALCUES del 2015 incorporaba un sistema de arbitraje de diferencias Estado-inversor caracterizado por la remisión a reglas internacionales en aspectos como el nombramiento de los árbitros o la transparencia del procedimiento arbitral, por hacer especial énfasis en la fase de consultas, y por adoptar un sistema procesal de no U-turn. Sin embargo, este régimen de arbitraje de inversiones cambió por completo con el borrador del APIUES del 2018, donde se sustituye el arbitraje de diferencias Estado-inversor por un mecanismo de STI. La presente investigación fundamenta esta evolución en dos argumentos mayores.

${ }^{74}$ STJUE, de 6 de marzo de 2018, Achmea, C-284/16, EU:C:2018:158, 58.

75 Tratado de Funcionamiento de la Unión Europea (TFUE), de 25 de marzo de 1957 (DOUE, n. ${ }^{\circ} \mathrm{C} 202$ de 7 de junio de 2016).

${ }^{76}$ STJUE, de 6 de marzo de 2018, Achmea, C-284/16, EU:C:2018:158, 61.

77 Hess, The Fate of Investment Dispute Resolution after the Achmea Decision of the European Court of Justice, 9-10, traducción de la autora. 
En primer lugar, si bien el borrador del APIUES mantiene la preocupación por aspectos del borrador del ALCUES como la transparencia o el establecimiento de un código de conducta para los árbitros, el APIUES introduce cambios sustanciales en tanto que aporta mayor claridad y especialidad a ese régimen anterior mediante un desarrollo normativo mucho más extenso, cambiando desde aspectos formales como la duración de los plazos en la fase de consultas a cuestiones de fondo como la composición de los Tribunales. Relacionado con esta observación está el segundo argumento. Los cambios introducidos por el borrador del APIUES otorgan a este acuerdo muchas de las características básicas del STI implementado en la ATCI y el AECG. En particular, destaca el cambio en la composición del tribunal arbitral en tanto que el APIUES sustituye un sistema donde las partes eligen a los árbitros de un único tribunal por un sistema donde los árbitros son elegidos por las partes separada y conjuntamente, y estos árbitros pueden pertenecer a un Tribunal de Primera Instancia o un Tribunal de Apelaciones, el último de carácter internacional y permanente (ver epígrafe IV.1).

Las razones que han llevado a este cambio en el sistema de arbitraje de inversiones tienen que ver con la incorporación del STI en otros ALCs de la Unión, como la ATCI o el AECG (ver epígrafe II). No obstante, cabe preguntarse si los desafíos legales del sistema de arbitraje de diferencias Estadoinversor a los que ha tenido que dar respuesta el TJUE en el Dictamen 2/15 o en el caso Achmea han podido influir también en la decisión de la Unión de cambiar el sistema de arbitraje del borrador del Capítulo IX del ALCUES al reflejado en el Capítulo III del borrador del APIUES.

Además, resulta de interés para análisis futuros seguir la evolución de la propuesta de la Comisión Europea de mayo del 2015, recogida en el documento «Investment in TTIP and beyond - the path for reform - Enhancing the right to regulate and moving from current ad hoc arbitration towards an Investment Court», de establecer un Tribunal Multilateral de Inversiones una vez superada la primera fase del $\mathrm{STI}^{78}$. La diferencia básica entre un STI y un Tribunal Multilateral de Inversiones se encuentra en el ámbito de aplicación de cada uno: mientras el STI se aplicaría únicamente a los ALCs de la UE con países terceros, el Tribunal Multilateral de Inversiones tendría un alcance global y afectaría en principio incluso a los APPRIs intra-UE ${ }^{79}$. Sin embargo, cabe cuestionar esta última declaración, pues la CE ha solicitado este año a

${ }^{78}$ «Investment in TTIP and beyond - the path for reform Enhancing the right to regulate and moving from current ad hoc arbitration towards an Investment Court», Comisión Europea, acceso el 12 de mayo de 2017.

79 «State of the Union - A Multilateral Investment Court», Comisión Europea, acceso el 24 de abril de 2018. 
los Estados Miembros de la UE que terminen los 196 APPRIs intra-UE existentes actualmente (ver epígrafe V.2).

En el caso del APIUES, el artículo 3.12 del Acuerdo obliga a las partes y otros socios comerciales interesados «a intentar alcanzar el establecimiento de un tribunal multilateral de inversiones y un mecanismo de apelación para la resolución de conflictos sobre inversiones», pero no hay ningún indicio suplementario de un posible Tribunal Multilateral de Inversiones. Terminamos pues este análisis con una mirada al futuro y la siguiente pregunta: ¿serán nuevos cambios necesarios en el APIUES para adaptar el acuerdo a esa segunda fase del proyecto europeo en materia de arbitraje de inversiones?

Desde mi punto de vista, no creo que la introducción de un Tribunal Multilateral de Inversiones suponga cambios mayores para el APIUES y ello por dos razones principales. En primer lugar, el régimen de arbitraje previsto bajo el Tribunal de Inversiones no difiere sustancialmente del STI, pues ambos mecanismos pretenden establecer un «marco de solución de diferencias internacionales en materia de inversiones que sea permanente, independiente y legítimo; previsible por su jurisprudencia reiterada; que permita apelar las decisiones; eficaz en función de los costos; dotado de procedimientos transparentes y eficaces $\rangle^{80}$. En segundo lugar, me parece un proyecto difícil de alcanzar en sí, pues requiere obtener la participación y el consentimiento de la comunidad internacional para lograr ese alcance global.

\section{BIBLIOGRAFÍA}

\section{Normativa}

Convenio de 23 de mayo de 1969 de las Nacionales Unidas sobre el Derecho de los Tratados, hecho en Viena. Instrumento de adhesión de España de 2 de mayo de 1972 (BOE n. ${ }^{\circ} 142$ de 13 de junio de 1980)

Convenio de 18 de marzo de 1965 sobre Arreglo de Diferencias Relativas a Inversiones entre Estados y nacionales de otros Estados, hecho en Washington. Instrumento de adhesión de España de 20 de junio de 1994, (BOE n. ${ }^{\circ} 219$ de 13 de septiembre de 1994). Para acceder a la lista completa de los Estados que han ratificado la Convención: https://icsid.worldbank.org/en/Documents/icsiddocs/ List $\% 20$ of $\% 20$ Contracting $\% 20$ States $\% 20$ and $\% 20$ Other $\% 20$ Signatories $\% 20$ of\%20the\%20Convention\%20-\%20Latest.pdf (acceso el 20 de abril de 2018).

${ }^{80}$ Recomendación, de 13 de septiembre de 2017, de la Comisión Europea de Decisión del Consejo por la que se autoriza la apertura de negociaciones sobre un Convenio relativo al establecimiento de un tribunal multilateral para la solución de diferencias en materia de inversiones, hecha en Bruselas. Eur-lex, n. ${ }^{\circ}$ 2017/0493, 2. 
Ley Modelo de la Comisión de las Naciones Unidas para el Derecho Mercantil Internacional sobre Arbitraje Comercial Internacional, de 21 de junio de 1985, con las enmiendas aprobadas en el 2006. Nueva York, 2008.

El texto se puede consultar en la página oficial de la CNUDMI: http://www.uncitral. org/uncitral/es/uncitral_texts/arbitration/1985Model_arbitration.html

Tratado de Funcionamiento de la Unión Europea (TFUE), de 25 de marzo de 1957, (DOUE n. ${ }^{\circ} \mathrm{C} 202$ de 7 de junio de 2016)

Comisión Europea, Recomendación de Decisión del Consejo por la que se autoriza la apertura de negociaciones sobre un Convenio relativo al establecimiento de un tribunal multilateral para la solución de diferencias en materia de inversiones, 13 de septiembre de 2017, COM (2017) 493 final.

Recomendación de 13 de septiembre de 2017 de la Comisión Europea de Decisión del Consejo por la que se autoriza la apertura de negociaciones sobre un Convenio relativo al establecimiento de un tribunal multilateral para la solución de diferencias en materia de inversiones, hecha en Bruselas. Eur-lex, n. ${ }^{\circ}$ 2017/0493.

\section{Normativa internacional en fase de proyecto}

Convención de 21 de marzo de 1986 de Viena sobre el Derecho de los Tratados celebrados entre Estados y Organizaciones Internacionales. Para acceder al borrador y a la lista completa de los Estados que han ratificado la Convención: https:// treaties.un.org/Pages/ViewDetails.aspx?src=TREATY\&mtdsg_no=XXIII$3 \&$ chapter $=23 \&$ lang $=$ en

Acuerdo de abril del 2018 de Libre Comercio entre la Unión Europea y Singapur. Borrador. http://trade.ec.europa.eu/doclib/press/index.cfm?id=961 (acceso el 22 de abril de 2018).

Acuerdo de abril del 2018 de Protección de las Inversiones entre la Unión Europea y Singapur. Borrador. Caps. III y IV, Anexo 7 y Anexo 8. http://trade.ec.europa.eu/ $\mathrm{doclib} /$ press/index.cfm?id=961 (acceso el 22 de abril de 2018).

Acuerdo de mayo del 2015 de Libre Comercio entre la Unión Europea y Singapur. Borrador.

Cap. IX: http://trade.ec.europa.eu/doclib/docs/2014/october/tradoc_152844.pdf (acceso el 17 de abril de 2018).

Anexo 9-F: http://trade.ec.europa.eu/doclib/docs/2015/june/tradoc_153580.pdf (acceso el 17 de abril de 2018).

Anexo 9-G: http://trade.ec.europa.eu/doclib/docs/2015/june/tradoc_153581.pdf (acceso el 17 de abril de 2018).

Convención de 21 de marzo de 1986 sobre el Derecho de los Tratados celebrados entre Estados y Organizaciones Internacionales (CVDTEOI), hecha en Viena. http://legal.un.org/ilc/texts/instruments/english/conventions/1_2_1986.pdf (acceso el 21 de abril de 2018).

\section{Jurisprudencia}

STJUE de 6 de marzo de 2018, Achmea, C-284/16, EU:C:2018:158. 
Conclusiones del Abogado General Sr. Melchior Wathelet de 19 de septiembre de 2017, Achmea, C-284/16, EU:C:2017:699.

Dictamen del Pleno del TJUE de 16 de mayo de 2017, C-2/15, EU:C:2017:376.

Conclusiones de la Abogado General Sra. Eleanor Sharpston de 21 de diciembre de 2016, C-2/15, EU:C:2016:992.

\section{Doctrina}

ADVISORY COUNCIL ON INTERNATIONAL AFFAIRS. «Introduction». En International Investment Dispute Settlement, . $^{\circ}$ 95, 7-12. La Haya: Advisory Council on International Affairs, 2015.

Borovikov, E.; Evtimov, B. y Crevon-Tarassova, A. «European Union». En The International Arbitration Review, ed. por James H. Carter, Vol. 7, 186-195. Londres: Law Business Research Ltd, 2016. https://thelawreviews.co.uk//digital_assets/a89cacdf-f2e2-4e82-9707-2411fd3991f0/The-International-Arbitration-Review-7th-ed.pdf (acceso el 29 de marzo de 2018).

Cremona, M. «Shaping EU Trade Policy post-Lisbon: Opinion 2/15 of 16 May 2017». En European Constitutional Law Review, ed. por Leonard F. M. Besselink, Monica Claes y Jan-Herman Reestman, Vol. 14, 231-259. Cambridge: Cambridge University Press, 2018. https:/www.cambridge.org/core/journals/ european-constitutional-law-review/article/shaping-eu-trade-policy-postlisbonopinion-215-of-16-may-2017/F04F045C4F26BF07455E207C25E6E9A3 (acceso el 3 de abril de 2018).

De La Villa GiL, L. E. «El futuro de la Unión Europea. Declaración de Laeken». En Revista del Ministerio de Trabajo y Asuntos Sociales, n. ${ }^{\circ}$ 37, 221-229. 2001. http://www.empleo.gob.es/es/publica/pub_electronicas/destacadas/revista/numeros/37/docu1.pdf (acceso el 26 de abril de 2018).

ELMS, D. «Understanding the EU-Singapore Free Trade Agreement». En Australia, the European Union and the New Trade Agenda, ed. por Annmarie Elijah, Donald Kenyon, Karen Hussey y Pierre Van Der Eng, 35-54. Canberra: Australian National University Press, 2017. http://www.oapen.org/download?type=docume nt\&docid=633015 (acceso el 29 de marzo de 2018).

HaRte, R. y PuCCIO, L. From arbitration to the investment court system (ICS) - The evolution of CETA rules, 5-20. Servicios De Estudios Del Parlamento Europeo (EPRS), 2017.http://www.europarl.europa.eu/RegData/etudes/IDAN/2017/607251/ EPRS_IDA(2017)607251_EN.pdf (acceso el 29 de marzo de 2018).

Hess, B. The Fate of Investment Dispute Resolution after the Achmea Decision of the European Court of Justice, n. ${ }^{\text {o } 3, ~ 5-20 . ~ L u x e m b u r g o: ~ M a x ~ P l a n c k ~ I n s t i t u t e ~ L u-~}$ xembourg for Procedural Law Research Paper Series, 2018. http://www.mpi.lu/ fileadmin/mpi/medien/events/2018/4/3/WPS3_2018_The_Fate_of_Investment Dispute_Resolution_after_the_Achmea_Decision_of_the_European_Court_of_ Justice (acceso el $3 \overline{\text { de }}$ abril de 2018 ).

Hindelang, S. y Hagemeyer, T. M. In Pursuit of an International Investment Court: Recently negotiated investment chapters in EU Comprehensive FTA in comparative perspective, 1-215. Parlamento Europeo, Directorate-General for External Poli- 
cies, 2017. http:/www.europarl.europa.eu/RegData/etudes/STUD/2017/603844/ EXPO_STU(2017)603844_EN.pdf (acceso el 29 de marzo de 2018).

Hindelang, S. y SASSENRATH, C.-P. The investment chapters of the EU's international trade and investment agreements in a comparative perspective, 1-173. Parlamento Europeo, Directorate-General for External Policies, 2015. http://www.europarl.europa.eu/RegData/etudes/STUD/2015/534998/EXPO_STU(2015)534998_EN.pdf (acceso el 29 de marzo de 2018).

HsU, L. «EU-ASEAN trade and investment relations with special focus on Singapore». En European Yearbook of International Economic Law, ed. por Christoph Herrmann, Markus Krajewski y Jörg Philipp Terhechte, Vol. 6, 233-250. Springer, 2015. http://ink.library.smu.edu.sg/cgi/viewcontent.cgi?article $=4487 \&$ context $=$ sol_research (acceso el 29 de marzo de 2018).

Kleimann, D. «Beyond the Shadow of the Veto: Economic Treaty-Making inthe European Union after Opinion 2/15». En Institutionalisation Beyond the Nation State. Series Studies in European Economic Law and Regulation, ed. por Élaine Fahey, Vol. 10, 1-24. Cham: Springer, 2018. https://www.academia.edu/36433589/Beyond the_Shadow_of the Veto_Economic_Treaty-Making_in_the_European Union_after_Opinion_- 2 _15 (acceso el 26 de abril de 2018).

LAVRanos, N. «In-depth briefing: Mixed exclusivity - The Court of Justice of the EU's Opinion on the EU Singapore FTA», 1-8. Borderlex, 2017. http://borderlex. eu/wp-content/uploads/2017/05/2017-05-18-IN-DEPTH-REPORT-BORDERLEX-SINGAPORE-FTA.pdf (acceso el 29 de marzo de 2018).

LATEK, M. Investor-State Dispute Settlement (ISDS) State of play and prospects for reform, 1-9. Servicios De Estudios Del Parlamento Europeo (EPRS), 2014. http:// www.europarl.europa.eu/RegData/bibliotheque/briefing/2014/130710/LDM BRI\%282014\%29130710_REV2_EN.pdf (acceso el 25 de abril de 2018).

LÓPEZ BofILl, H. «Change in the Constitutional Framework of the European Union with regard to regulating foreign direct investment». En Revista Catalana de Dret Públic, Vol. 51, 104-121. 2015. https://dialnet.unirioja.es/descarga/articulo/5733166.pdf (acceso el 29 de marzo de 2018).

NORTON ROSE FULBRIGHT. «CJEU rules on EU's proposed free trade agreement with Singapore». 2017. http://www.nortonrosefulbright.com/knowledge/publications/149650/cjeu-rules-on-eus-proposed-free-trade-agreement-with-singapore (acceso el 3 de abril de 2018).

PAscuAl ViVEs, F. «El futuro del arbitraje de inversión en los acuerdos internacionales celebrados por la Unión Europea». En Revista Electrónica de Estudios Internacionales, n. ${ }^{\circ}$ 33, 2-35. 2017. http://www.reei.org/index.php/revista/num33/archivos/02_Estudio_PASCUAL_Francisco.pdf (acceso el 29 de marzo de 2018).

Roussou, E. Free Trade Agreement between the EU and the Republic of Singapore - Analysis, 1-102. Parlamento Europeo, Directorate-General for External Policies, 2018 (acceso el 19 de abril del 2017).

Segura Serrano, A. «El Acuerdo de Libre Comercio entre la UE y Canadá (CETA): Una evaluación de la política comercial de la UE». En Revista Electrónica de Estudios Internacionales, n. ${ }^{\circ}$ 30, 2-22. 2015. https://dialnet.unirioja.es/descarga/ articulo/5335862.pdf (acceso el 29 de marzo de 2018). 
Urrea Corres, M. «La política (común) de seguridad y defensa en el Tratado de Lisboa: La eficacia como objetivo, la flexibilidad como instrumento y la ambición como propuesta». En Revista Española de Derecho Europeo, n. ${ }^{\circ}$ 33, 1-18. Pamplona: Editorial Civitas, SA, 2010.

Zestos, G. K. y Coffman, C. W. «Transatlic Economic Integration». En The TTIP - The Transatlantic Trade and Investment Partnership between the European Union and the United States, ed. por Joaquín y Roberto Domínguez, 73. Miami: Miami-Florida European Union Center of Excellence, 2014.

\section{Otros}

ALLEN, R. «EU Court thwarts prompt ratification of EU-Singapore Free Trade Agreement». Global Arbitration News (2017). https://globalarbitrationnews.com/eucourt-thwarts-prompt-ratification-eu-singapore-free-trade-agreement/ (acceso el 3 de abril de 2018).

Comisión EuropeA. «EU-Singapore Trade \& Investment». Acceso el 19 de abril de 2018. https://eeas.europa.eu/sites/eeas/files/9045-trade-eu-singapore-trade-booklet-170630.pdf.

«Guide to the EU-Singapore Free Trade Agreement and Investment Protection Agreement». Acceso el 19 de abril de 2018. http://trade.ec.europa.eu/doclib/ docs/2018/april/tradoc_156711.pdf.

— «Next steps towards finalising the EU-Singapore agreements». Acceso el 19 de abril de 2018. http://trade.ec.europa.eu/doclib/docs/2018/april/tradoc_156716. pdf.

—El AECG explicado». Acceso el 13 de abril de 2018. http://ec.europa.eu/trade/ policy/in-focus/ceta/ceta-explained/index_es.htm.

— «State of the Union - A Multilateral Investment Court». Acceso el 24 de abril de 2018. http://trade.ec.europa.eu/doclib/docs/2017/september/tradoc_156042.pdf.

- «Investment in TTIP and beyond - the path for reform Enhancing the right to regulate and moving from current ad hoc arbitration towards an Investment Court». Acceso el 12 de mayo de 2017. http://trade.ec.europa.eu/doclib/ docs $/ 2015 /$ may/tradoc_153408.PDF.

— «La Comisión propone un nuevo Sistema de Tribunales de Inversiones para la ATCI y para otras negociaciones comerciales y de inversión de la UE». Acceso el 25 de abril de 2018. http://europa.eu/rapid/press-release_IP-15-5651_es.htm.

CONSEJO DE LA UNIÓN EUROPEA. «Desclasificación parcial del documento 17494/09 RESTREINT UE». Acceso el 17 de abril de 2018. http://data.consilium.europa. eu/doc/document/ST-17494-2009-EXT-1/es/pdf.

Cortes Generales. «Diario de Sesiones del Congreso de los Diputados». Acceso el 21 de abril de 2018. http://www.congreso.es/public_oficiales/L10/CONG/DS/ CO/DSCD-10-CO-832.PDF. 


\title{
EL ARBITRAJE DE INVERSIONES ENTRE LA UNIÓN EUROPEA Y SINGAPUR: ¿DEL ARBITRAJE DE DIFERENCIAS ESTADO-INVERSOR A UN SISTEMA DE TRIBUNALES DE INVERSIONES?
}

\section{Investment arbitration between the European Union and Singapore: from the Investor-State Dispute Settlement to an Investment Court System?}

\author{
Sophie Recio San Emeterio \\ Universidad de Deusto \\ sophie.recio@opendeusto.es
}

http://dx.doi.org/10.18543/ed-67(1)-2019pp353-383

\section{Copyright}

Estudios de Deusto es una revista de acceso abierto, lo que significa que es de libre acceso en su in tegridad. Se permite su lectura, la búsqueda, descarga, distribución y reutilización legal en cualquier tipo de soporte sólo para fines no comerciales, sin la previa autorización del editor o el autor, siempre que la obra original sea debidamente citada y cualquier cambio en el original esté claramente indicado

Estudios de Deusto is an Open Access journal which means that it is free for full access, reading, search, download, distribution, and lawful reuse in any medium only for non-commercial purposes, without prior permission from the Publisher or the author; provided the original work is properly cited and any changes to the original are clearly indicated. 